\title{
Overlapping promoters modulate Fnr- and ArcA-dependent anaerobic transcriptional activation of the focApfl operon in Escherichia coli
}

\author{
Manuela Kaiser ${ }^{1}$ and Gary Sawers ${ }^{2}$
}

Author for correspondence: Gary Sawers. Tel: +441603456900 ext. 2750. Fax: +441603454970 . e-mail: gary.sawers@bbsrc.ac.uk

1 Lehrstuhl für Mikrobiologie, der Universităt München, Maria-Ward-Straße 1a, D-80638 Munich, Germany

2 Nitrogen Fixation Laboratory, John Innes Centre, Norwich NR4 7UH, UK

\begin{abstract}
The recently identified P6A promoter of the anaerobically inducible focApfI operon of Escherichia coli overlaps the Fnr (fumarate-nitrate reduction regulator)-dependent P6 promoter. The Fnr-binding site of P6 and the -35 hexamer sequence of $P 6 A$ are shared between the promoters. Inactivation of P6A, through introduction of a -10 hexamer mutation, resulted in enhanced anaerobic induction of operon expression. The dependence on the ArcA (aerobic respiration control regulator) and Fnr transcription factors for anaerobic induction was tested for several focA-lacZ and pfl-lacZ gene fusions. Anaerobic induction became more dependent on Fnr in derivatives lacking a functional P6A promoter compared with wild-type constructs. Moreover, aerobic expression of the focA gene was reduced by the p6A mutation, as was the dependence on ArcA for anaerobic induction. Inactivation of P6 severely reduced Fnr-dependent anaerobic induction, in accord with previous findings. Transcription analyses demonstrated that a mutation in the $\mathbf{- 1 0}$ hexamer sequence of either P6A or P6 did not adversely affect transcription from the remaining promoter. Taken together, these results indicate that the $P 6 A$ promoter moderates the Fnr-dependent activation of $\mathbf{P 6}$ through competition for RNA polymerase binding.
\end{abstract}

Keywords: gene regulation, transcription factors, overlapping promoters, Fnr and ArcA, anaerobic metabolism

\section{INTRODUCTION}

The gene encoding the enzyme pyruvate formate-lyase (Pfl) forms an operon with a gene termed focA (Sawers \& Böck, 1988, 1989; Suppmann \& Sawers, 1994). Pfl is the central enzyme of anaerobic carbon metabolism in Escherichia coli and catalyses the non-oxidative, coenzyme A-dependent cleavage of pyruvate to formate (Knappe \& Sawers, 1990). The product of the focA gene is an integral membrane protein required to modulate the intracellular formate pool (Suppmann \& Sawers, 1994). The focApfl operon is expressed at low levels in aerobic cells but upon oxygen depletion, expression is induced between 10- and 15-fold (Sawers \& Böck, 1988, 1989). Anaerobic induction of expression is controlled by the two global transcription factors ArcA (aerobic

Abbreviations: ArcA, aerobic respiration control regulator; Fnr, fumarate-nitrate reduction regulator; Pfl, pyruvate formate-lyase. respiration control regulator) and Fnr (fumarate-nitrate reduction regulator) (Sawers \& Suppmann, 1992; Sawers, 1993; Drapal \& Sawers, 1995a, b; Kaiser \& Sawers, 1995).

Transcription of the focApfl operon is directed by multiple promoters (Sawers \& Böck, 1989; Sawers, 1993). Five promoters are located within the operon and lie directly upstream of the $p f l$ gene (Fig. 1). A further three promoters are located in an approximately $500 \mathrm{bp}$ regulatory region immediately upstream of the foc $A$ gene. One of these upstream promoters, P6A, was discovered in a recent study which examined in vitro and in vivo expression from the Fnr-dependent $P 6$ promoter (Kaiser \& Sawers, 1995). P6 and P6A overlap and their respective initiation sites are separated by $10 \mathrm{nt}$, which indicates that the promoters are separated by one helical turn. The P6 promoter has a poorly conserved -10 hexamer sequence and lacks a -35 hexamer sequence. The $P 6$ promoter does, however, have a 


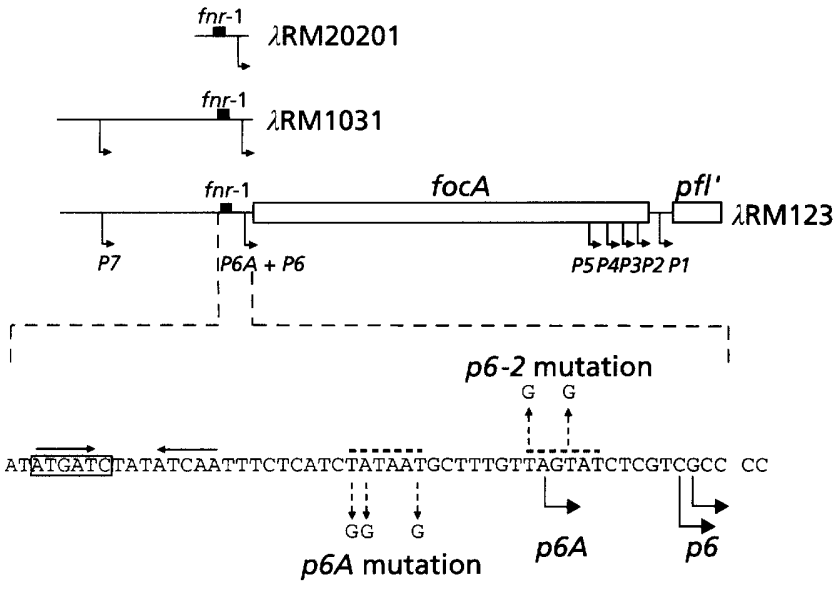

Fig. 1. Schematic representation of the focApfl operon promoter-regulatory region. The upper portion of the figure shows the focApfl operon promoter-regulatory region, the focA gene and the $5^{\prime}$ part of the pfl gene. The DNA fragments used to construct the various focApfl promoter-lacZ gene fusions are designated $\lambda R M 20201, \lambda R M 1031$ and $\lambda R M 123$. The lower portion of the figure shows the DNA sequence of the 'top' strand of the $P 6$ and $P 6 A$ promoters. The more $3^{\prime}$ transcription initiation site of the $P 6$ promoter is designated +1 . The -10 hexamer sequences of the $P 6$ and $P 6 A$ promoters are overlined and the mutations introduced into the respective hexamer are shown by vertical, dashed arrows. The -35 hexamer sequence of the P6A promoter is boxed and the palindromic $\mathrm{Fnr}$ recognition sequences are highlighted by converging horizontal arrows. fnr- 1 indicates that the Fnrbinding site is mutated.

palindromic Fnr-binding site (Spiro \& Guest, 1990) which is centred at -41.5 bp relative to the transcription initiation site and directs anaerobic, Fnr-dependent transcription (Kaiser \& Sawers, 1995). The P6A promoter, in contrast, has a consensus -10 hexamer sequence and a -35 hexamer sequence that matches the consensus in three positions (Fig. 1). Consequently, transcription from the $P 6$ promoter is regulated by Fnr, whilst transcription from P6A appears to be factorindependent (Kaiser \& Sawers, 1995). The relationship between these promoters is complicated by the fact that the upstream 'half-site' of the Fnr recognition sequence and the -35 hexamer directly overlap. This has major implications for the functions of these promoters in controlling focApfl operon expression. The aim of this study therefore was to characterize the $P 6 A$ promoter in an attempt to identify its physiological function. Through the use of specific mutagenesis of the -10 hexamer sequences we have inactivated each promoter individually. These studies have provided credence for the hypothesis that an important function of $P 6 A$ is to modulate transcription initiation from $P 6$ through competition between RNA polymerase and the Fnr transcription factor for a common binding site.

\section{METHODS}

Strains, plasmids, phages and growth conditions. Bacterial strains, plasmids and phages used in this study are listed in Table 1. Bacteria were grown in buffered rich medium as described by Begg et al. (1977). Glucose was added to a final concentration of $20 \mathrm{mM}$ for both aerobic and anaerobic growth and cells for both enzyme assay and RNA preparation were harvested in the mid-exponential phase of growth. Chloramphenicol was used at a concentration of $15 \mu \mathrm{g} \mathrm{ml}^{-1}$ while kanamycin was used at $50 \mu \mathrm{g} \mathrm{ml}^{-1}$. Media and buffers used for $\lambda$ work were prepared as described by Sawers \& Böck (1989).

Plasmid construction. The construction of pRS551 and pRS552 derivatives pRM23, pRM2307, pRM2317, pRM1031 and pRM20201, which harbour various portions of wild-type and mutant derivatives of the $p f l$ regulatory region fused with the lacZ gene, has been described previously (Sawers \& Böck, 1988; Sawers, 1993; Drapal \& Sawers, 1995a; Kaiser \& Sawers, 1995). These constructs were used directly or acted as templates for the construction of further mutant derivatives by PCR amplification with the appropriately designed oligonucleotides. Transfer of $p f l-l a c Z$ fusions to the specialized $\lambda$ transducing phage $\lambda$ RS45 was performed as described by Simons et al. (1987). The various promoter mutations had no influence on translation of either the $f \circ c A$ or $p f l$ genes, thus operon and protein fusions gave comparable results.

Primer extension analysis of total RNA. Total RNA was isolated from exponentially growing cells using the RNeasy kit (Qiagen) according to the manufacturer's instructions. Primer extension analysis of $p f l$ P6- and P6A-derived transcripts was performed with $10 \mu \mathrm{g}$ total RNA and $0.2 \mathrm{pmol}^{32} \mathrm{P}$ end-labelled primer according to Sawers \& Böck (1989). Care was taken to ensure equal loading of RNA and the results of our primer extension analyses were highly reproducible. The oligonucleotides used in these studies included LACZ-1 $\left(5^{\prime}\right.$ GGTCATAGCTGTTTCCTGTGTG $3^{\prime}$ ), which primes within the lacZ gene, and PFL16 (5' GTAAAAGATCAAAAGGGTTGTCAG $3^{\prime}$ ), which hybridizes downstream of the P6 promoter. Products of the primer extension reactions were separated on $6 \%$ polyacrylamide sequencing gels containing $7 \mathrm{M}$ urea.

$\beta$-Galactosidase enzyme assays. $\beta$-Galactosidase enzyme activity was determined in cultures of exponentially growing cells, and the specific activity was calculated according to Miller (1972). Values reported are the mean of at least three independent experiments performed in triplicate. The SEM of the values reported was not more than $15 \%$.

Other methods. Densitometric quantification of autoradiograms was performed using a Mirror 600 Plus Scanner and $\mathrm{MacBas}$ version 2.0 software. Several exposures of film were used to ensure that data were collected in the linear sensitivity range of the film.

\section{RESULTS}

\section{Influence of -10 hexamer mutations on $P 6$ and $P 6 A$ transcription}

The locations of the transcription initiation sites of the $P 6$ and P6A promoters have been determined both in vitro and in vivo (Sawers \& Böck, 1989; Sawers, 1993; Kaiser \& Sawers, 1995). These initiation sites are separated by $10 \mathrm{bp}$ (Fig. 1). In an attempt to characterize the relationship between these two promoters and their influence on $p f l$ expression, -10 hexamer mutations, which inactivate the promoters, were introduced into the three focA-lacZ fusion constructs $\lambda R M 20201$, $\lambda$ RM1031 and $\lambda$ RM123 (Fig. 1). The p6-2 mutation was introduced into the P6 - 10 hexamer and the $p 6 \mathrm{~A}$ 
Table 1. Strains, plasmids and phages used in this study

\begin{tabular}{|c|c|c|}
\hline $\begin{array}{l}\text { Strain, plasmid } \\
\text { or phage }\end{array}$ & Genotype* & Reference or source \\
\hline \multicolumn{3}{|l|}{ Strain } \\
\hline MC4100 & $\mathrm{F}^{-}$araD139 $\Delta(\arg F-l a c) U 169$ ptsF25 deoC1 relA1 flbB530 rpsL150 $\lambda^{-}$ & Casadaban \& Cohen (1979) \\
\hline RM102 & Like MC4100 but $\Delta f n r \Delta(\operatorname{srl}-r e c A) 306:: \operatorname{Tn} 10$ & Birkmann et al. (1987) \\
\hline RM313 & Like MC4100 but arcA1 zjj::Tn10 & Sawers \& Suppmann (1992) \\
\hline RM315 & Like MC4100 but $\Delta f n r \operatorname{arcA1} z j j:: \operatorname{Tn} 10$ & Sawers \& Suppmann (1992) \\
\hline RM2307 & Like MC4100 but carrying the $p 6 A$ mutation in the $p f l$ regulatory region & Sawers (1993) \\
\hline \multicolumn{3}{|l|}{ Plasmid } \\
\hline pRS551 & $\mathrm{Km}^{\mathrm{R}} \mathrm{Ap}^{\mathrm{R}}$ lacZ lacY lacA & Simons et al. (1987) \\
\hline pRM23 & pRS552 $\Phi\left(p f l^{\prime}-l a c Z\right) 1397(\mathrm{Hyb})$ & Sawers \& Böck (1988) \\
\hline pRM2307 & $\begin{array}{l}\text { Like pRM } 23 \Phi\left(p f l^{\prime}-l a c Z\right) 1397(\mathrm{Hyb}) \text { but carrying the } p 6 A-10 \text { hexamer } \\
\text { mutation }\end{array}$ & Sawers (1993) \\
\hline pRM2317 & $\begin{array}{l}\text { Like pRM23 } \Phi\left(p f^{\prime}-l a c Z\right) 1397(\mathrm{Hyb}) \text { but carrying the } p 6-2-10 \text { hexamer } \\
\text { mutation }\end{array}$ & Sawers (1993) \\
\hline pRM1031 & $\begin{array}{l}\text { pRS551 } \Phi\left(f o c A^{\prime}-' \text { lac } Z\right) \text { carrying the } 493 \text { bp insert from the focApfl regulatory } \\
\text { region }\end{array}$ & Drapal \& Sawers (1995a) \\
\hline pRM1036 & Like pRM1031 but carrying the $p 6-2$ mutation & This work \\
\hline pRM1036A & Like pRM1031 but carrying the $p 6 \mathrm{~A}$ mutation & This work \\
\hline pRM20201 & $\begin{array}{l}\text { pRS551 } \Phi\left(f o c A^{\prime}-l a c Z\right) \text { carrying the } 119 \text { bp DNA insert from the focApfl } \\
\text { regulatory region }\end{array}$ & Kaiser \& Sawers (1995) \\
\hline pRM20203 & Like pRM20201 but carrying the $p 6-2$ mutation & This work \\
\hline pRM20203A & Like pRM20201 but carrying the $p 6 A$ mutation & This work \\
\hline $\begin{array}{l}\text { Phage } \\
\lambda \text { RS45 }\end{array}$ & lac $Z^{\prime} l a c Y ~ i m m^{21}$ ind & Simons et al. (1987) \\
\hline
\end{tabular}

*Ap, ampicillin; Km, kanamycin.

mutation into the P6A -10 hexamer. The wild-type and mutant $l a c Z$ derivatives were introduced in single copy in to the chromosome of various E. coli strains and used to study transcription and expression.

Initially we determined the effects of the promoter mutations on $P 6$ and $P 6 A$ transcription in vivo. For this purpose total RNA from wild-type and $f n r$ mutant strains bearing the $\lambda$ derivatives $\lambda$ RM20201, $\lambda$ RM20203 (carrying the $p 6-2$ mutation) and $\lambda$ RM20203A (carrying the $p 6 A$ mutation) was isolated. These derivatives include only the P6 and P6A promoters and the Fnr recognition sequence (Fig. 1; Kaiser \& Sawers, 1995; Drapal \& Sawers, 1995b). Using a primer which specifically hybridized within the lac $Z$ coding sequence it was possible to determine directly the transcripts derived from the lac $Z$ fusions (Fig. 2a). A single initiation site for $P 6$ could be identified, which is identical to that found for the wild-type chromosomal promoter (Sawers \& Böck, 1989; Drapal \& Sawers, $1995 \mathrm{~b})$. We have noted that transcription always initiates from a single site (the labelled $G$ residue in Fig. 1) when short promoter region fragments are analysed and often initiates from two adjacent sites (the labelled $\mathrm{C}$ and $\mathrm{G}$ residues in Fig. 1) in constructs which include the complete promoter-regulatory region plus the downstream promoters, for example from $\lambda$ RM123 (see Fig. 3; Sawers \& Böck, 1989; Sawers, 1993).
Transcription from $P 6 A$ initiated primarily at a site located at $-11 \mathrm{bp}$ relative to the start site of $P 6$ (Fig. 2a). This start site also correlates with a site used by the promoter in its natural chromosomal location. A weaker signal initiating at a $G$ residue located at -14 bp was occasionally observed (Kaiser \& Sawers, 1995; Drapal \& Sawers, 1995b).

Primer extension analysis of $\lambda$ RM20201 in a wild-type genetic background demonstrated that transcription from $P 6$ was increased anaerobically, while that from $P 6 A$ was essentially unaffected by the cellular oxygen status (Fig. 2a). Quantification of the transcripts indicated that the combined level of $P 6$ plus $P 6 A$ transcription increased approximately threefold under anaerobic conditions (Fig. 2b). Analysis of transcription in a $f n r$ mutant revealed a drastic reduction in transcription from $P 6$ and abolition of anaerobic induction. This result is in accord with previous findings (Sawers, 1993; Kaiser \& Sawers, 1995). The residual transcription from $P 6$ observed in the $f n r$ mutant was due to lowlevel cAMP-receptor protein (Crp)-dependent initiation (G. Sawers, M. Kaiser, A. Sirko \& M. Freundlich, unpublished results).

The $f n r$ mutation resulted in a slight increase in $P 6 A$ transcription anaerobically and a more frequent use of the secondary initiation site at -14 bp both aerobically and anaerobically (Fig. 2a). The consequence of these 
(a)

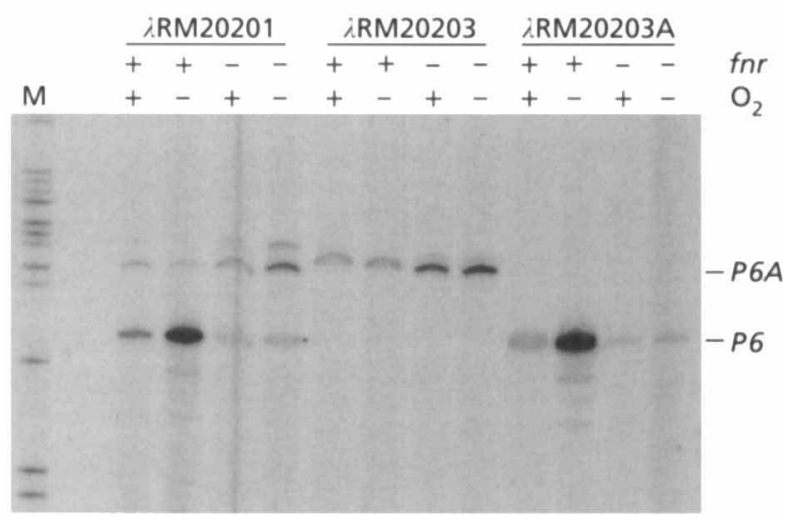

(b)

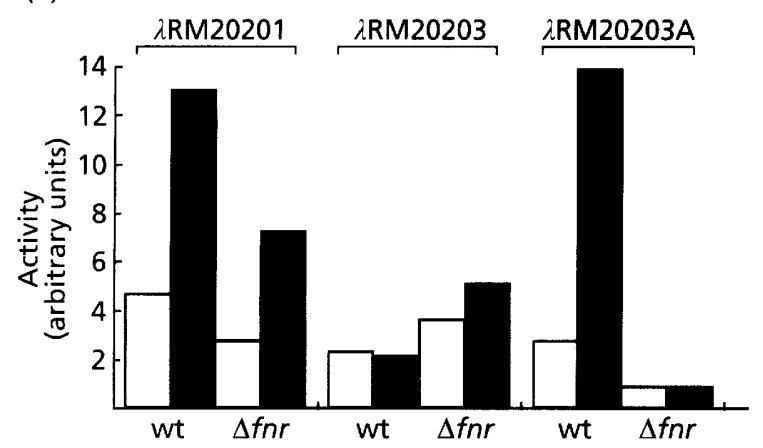

Fig. 2. Primer extension analysis of transcriptional lacZ fusions. (a) Total RNA was isolated from MC4100 and RM102 ( $\Delta$ fnr) carrying the focA-lacZ transcriptional fusions $\lambda R M 20201$ (wildtype $P 6$ and $P 6 A$ promoters), $\lambda$ RM20203 (P6 promoter mutant) or $\lambda$ RM20203A (P6A promoter mutant) after aerobic $\left(+\mathrm{O}_{2}\right)$ or anaerobic $\left(-\mathrm{O}_{2}\right)$ growth. Specific analysis of the transcripts generated by the fusions was achieved with an oligonucleotide which primed within the lacZ gene (see Methods). An autoradiogram is shown after the products were separated in a denaturing polyacrylamide gel. $\mathrm{M}$ designates the $\mathrm{C}$ reaction of a control DNA sequence. (b) Quantification of transcripts P6+P6A was performed after densitometric scanning of the autoradiogram shown in (a). The results presented show the total transcripts $(P 6+P 6 A)$ generated from the particular fusion. Open and filled bars represent analysis of RNA from aerobically and anaerobically grown cells, respectively. Strain MC4100 is designated as wt while strain RM102 is designated $\Delta f n r$.

slight alterations in $P 6 A$ transcription to the overall transcription from $\lambda \mathrm{R} M 20201$ was an approximately twofold reduction in transcription compared with the wild-type both aerobically and anaerobically; however, a 2.5 -fold anaerobic induction of transcription was still observed (Fig. 2b).

Fig. 2(a) shows that introduction of the $p 6-2$ mutation in derivative $\lambda$ RM20203 abolished transcription from the P6 promoter, as anticipated (Sawers, 1993). The p6-2 mutation had two consequences for P6A transcription: firstly, transcription initiated from the $G$ residue at position -12 in the mutant derivative, instead of the $A$ residue at $-11 \mathrm{bp}$; and secondly, the $G$ residue at $-14 \mathrm{bp}$ was no longer used as a site of initiation.
Interestingly, transcription initiated primarily from the A residue at $-11 \mathrm{bp}$ in a $f n r$ mutant both aerobically and anaerobically, indicating that Fnr can affect initiation from $P 6 A$ even when transcription initiation at $P 6$ is prevented by the $P 6-10$ hexamer. As was noted for $\lambda$ RM20201, the $f n r$ mutation resulted in enhanced P6A transcription; however, in the $\lambda$ RM20203 derivative the effect was observed both aerobically and anaerobically.

The $p 6 A$ mutation in $\lambda R M 20203 A$ completely inactivated the $P 6 A$ promoter while the regulation and levels of $P 6$ transcription remained unaffected (Fig. 2a). Anaerobic induction of $P 6$ transcription was $4 \cdot 6$-fold, while introduction of the $\mathrm{f} n \mathrm{r}$ mutation resulted in a $14-$ fold reduction in anaerobic transcription (Fig. 2b). A similar reduction in $P 6$ transcription was observed for the $\lambda$ RM20201 derivative but the overall effect on transcription was masked by transcription from $P 6 A$ (Fig. 2a, b).

\section{Effect of the $P 6$ and $P 6 A$ promoter mutations on expression from the $\lambda$ RM20201 focA-lacZ fusion derivatives}

The influence of chromosomal $\operatorname{arc} A$ and $f n r$ mutations on the levels of lacZ expression from $2 R M 20201$, $\lambda$ RM20203 and $\lambda$ RM20203A after aerobic and anaerobic growth was monitored (Table 2). Expression from $\lambda$ RM20201 was induced 3·4-fold by anaerobiosis, which is in good agreement with the transcription data (compare Fig. 2). Anaerobic induction was strongly reduced in a fnr mutant and was reduced to twofold by an $\operatorname{arc} A$ mutation. Whilst the $f n r$ mutation reduced the absolute level of anaerobic $\beta$-galactosidase enzyme activity but did not affect the aerobic activity level, the $\operatorname{arc} A$ mutation did not influence the anaerobic level of activity compared with the wild-type, but instead it resulted in a twofold increase in aerobic activity levels. This result is in agreement with the finding that ArcA has a negative influence on focA-lac $Z$ expression in this short promoter fragment (Drapal \& Sawers, 1995b). As expected from the transcription studies, the $p 6-2$ mutation in construct $\lambda$ RM20203 abolished anaerobic induction of expression (Table 2). Expression was derived solely from the activity of the P6A promoter (see Fig. 2a). ArcA had no major effect on $\beta$-galactosidase activity, whereas Fnr repressed expression anaerobically. Fnr did not show this effect in the absence of a functional ArcA protein.

The $p 6 A$ mutation on $\lambda$ RM20203A resulted in a threefold reduction in aerobic expression, indicating that the P6A promoter is responsible for the major proportion of the expression in aerobically grown cells (Table 2). Anaerobic growth resulted in a 13 -fold induction of expression which was absolutely dependent on the Fnr protein. An $\operatorname{arcA}$ mutation had little effect on expression; the mutation resulted in a slight increase in aerobic expression and a small decrease in anaerobic expression. These results demonstrate that the P6 promoter is responsible for the anaerobic induction of 
Table 2. Expression of the focA gene driven by the $P 6$ and $P 6 A$ promoters

\begin{tabular}{|c|c|c|c|c|}
\hline \multirow[t]{2}{*}{ lac $Z$ derivative ${ }^{*}$} & \multirow[t]{2}{*}{ Host genotype } & \multicolumn{3}{|c|}{$\beta$-Galactosidase activity (Miller units) } \\
\hline & & $\begin{array}{c}\text { Aerobic } \\
\left(+\mathrm{O}_{2}\right)\end{array}$ & $\begin{array}{c}\text { Anaerobic } \\
\left(-\mathrm{O}_{2}\right)\end{array}$ & $\begin{array}{c}\text { Induction } \\
\text { ratio } \\
\left(-\mathrm{O}_{2} /+\mathrm{O}_{2}\right)\end{array}$ \\
\hline \multirow[t]{4}{*}{$\lambda$ RM20201 (wild-type) } & $w t$ & 360 & 1215 & $3 \cdot 4$ \\
\hline & $\Delta f n r$ & 300 & 400 & $1 \cdot 3$ \\
\hline & $\operatorname{arcA}$ & 710 & 1470 & $2 \cdot 1$ \\
\hline & $\operatorname{arcA} \Delta f n r$ & 300 & 550 & $1 \cdot 8$ \\
\hline \multirow[t]{4}{*}{$\lambda$ RM20203 (P6 mutant) } & wt & 210 & 130 & $0 \cdot 6$ \\
\hline & $\Delta f n r$ & 220 & 245 & $1 \cdot 1$ \\
\hline & $\operatorname{arcA}$ & 275 & 250 & 0.9 \\
\hline & $\operatorname{arcA} \Delta f n r$ & 280 & 330 & $1 \cdot 2$ \\
\hline \multirow[t]{4}{*}{$\lambda \mathrm{RM} 20203 \mathrm{~A}(P 6 A$ mutant $)$} & wt & 100 & 1300 & $13 \cdot 0$ \\
\hline & $\Delta f n r$ & 74 & 60 & $0 \cdot 85$ \\
\hline & $\operatorname{arcA}$ & 135 & 1040 & $7 \cdot 8$ \\
\hline & $\operatorname{arcA} \Delta f n r$ & 100 & 82 & $0 \cdot 82$ \\
\hline
\end{tabular}

$*$ The $\lambda$ derivatives of the correspondingly named plasmids were inserted in single copy into the chromosome of the strains to be tested.

focA expression, while the $P 6 A$ promoter modulates the aerobic level of expression. It is also noteworthy that the absolute level of anaerobic expression in the wild-type promoter derivative and the $p 6 \mathrm{~A}$ mutant is similar although there is a significant difference in anaerobic expression in the fnr mutant. These results suggest that the effect of both promoters on expression is not additive and when $P 6$ transcription is prevented by the $f n r$ mutation, P6A expression becomes unimpeded. This agrees with the results of the transcription studies in Fig. $2(a)$.

\section{The influence on focA-lacZ expression of the addition of promoter $P 7$ regulatory sequences}

The $\lambda$ RM20201 focA-lacZ fusion includes only a portion of the focApfl operon regulatory sequences and lacks promoter P7 and sequences important for ArcAdependent regulation of expression. To examine the effect of the $p 6-2$ and $p 6 A$ mutations on expression directed by the complete upstream promoter-regulatory region of the operon, we constructed a series of lac $Z$ operon fusions based on the $\lambda$ RM1031 derivative (Fig. 1; Drapal \& Sawers, 1995a, b). The results presented in Table 3 show that addition of the $P 7$ promoter sequences resulted in a 7.3-fold anaerobic induction which was strongly dependent on ArcA and only partially dependent on Fnr. Introduction of the $p 6-2$ mutation reduced anaerobic induction twofold; however, the absolute level of anaerobic expression was reduced threefold compared with the wild-type promoter fragment. Aerobic expression was also reduced partially by the $p 6-2$ mutation. The $p 6-2$ mutation had a comparable effect on expression as that observed when the wild-type promoter-regulatory region was analysed in a $f n r$ mutant. The residual anaerobic induction was absolutely dependent on a functional ArcA protein and was partially dependent on Fnr; in the fnr mutant an approximately threefold anaerobic induction was still apparent but the absolute level of $\beta$-galactosidase activity in both aerobically and anaerobically grown cells was reduced threefold. Taken together, these findings indicate that promoter $P 7$ activity accounts for the anaerobic induction of expression in derivative $\lambda$ RM1036 and that ArcA mediates anaerobic induction when the Fnr-dependent P6 promoter is inactivated.

The $p 6 \mathrm{~A}$ mutation caused a threefold reduction in aerobic expression when compared with the wild-type grown under the same conditions (Table 3). This confirms the earlier observation that $P 6 A$ activity is in part largely responsible for the expression of the foc $A$ gene in aerobically grown cells. A $15 \cdot 5$-fold anaerobic induction of lacZ expression from the $\lambda \mathrm{RM} 1036 \mathrm{~A}$ derivative in the wild-type strain was observed. This anaerobic induction was almost completely dependent on Fnr. An $\operatorname{arcA}$ mutation had a minimal effect, reducing enzyme activity by $20 \%$ and $30 \%$ in aerobically and anaerobically grown cells, respectively, relative to enzyme activity levels in the wild-type (Table 3 ). In an arcA fnr double null mutant, anaerobic $\beta$-galactosidase activity was reduced 60 -fold compared with the wildtype. Inactivation of the $P 6 A$ promoter, therefore, severely impairs ArcA-dependent anaerobic induction of foc $A$ gene expression. Since anaerobic induction becomes almost exclusively Fnr-dependent, these findings indicate that P6A controls the degree to which Fnr and ArcA control anaerobic induction of focA expression. These data also indicate that the P6A promoter is important for ensuring substantial levels of aerobic foc $A$ gene expression. 
Table 3. Expression of the focA gene driven by the $P 6, P 6 A$ and $P 7$ promoters

\begin{tabular}{|c|c|c|c|c|}
\hline \multirow[t]{2}{*}{ lac $Z$ derivative } & \multirow[t]{2}{*}{ Host genotype } & \multicolumn{3}{|c|}{$\beta$-Galactosidase activity (Miller units) } \\
\hline & & $\begin{array}{c}\text { Aerobic } \\
\left(+\mathrm{O}_{2}\right)\end{array}$ & $\begin{array}{c}\text { Anaerobic } \\
\left(-\mathrm{O}_{2}\right)\end{array}$ & $\begin{array}{c}\text { Induction } \\
\text { ratio } \\
\left(-\mathrm{O}_{2} /+\mathrm{O}_{2}\right)\end{array}$ \\
\hline \multirow[t]{4}{*}{ גRM1031 (wild-type) } & wt & 500 & 3650 & $7 \cdot 3$ \\
\hline & $\Delta f n r$ & 440 & 1260 & $2 \cdot 8$ \\
\hline & $\operatorname{arc} A$ & 265 & 380 & $1 \cdot 4$ \\
\hline & $\operatorname{arc} A \Delta f n r$ & 245 & 260 & $1 \cdot 1$ \\
\hline \multirow[t]{4}{*}{ iRM1036 (P6 mutant) } & wt & 330 & 1050 & $3 \cdot 2$ \\
\hline & $\Delta f n r$ & 125 & 360 & $2 \cdot 9$ \\
\hline & $\operatorname{arcA}$ & 100 & 120 & $1 \cdot 2$ \\
\hline & $\operatorname{arcA} \Delta f n r$ & 120 & 155 & $1 \cdot 3$ \\
\hline \multirow[t]{4}{*}{$\lambda$ RM1036A (P6A mutant) } & wt & 160 & 2490 & $15 \cdot 5$ \\
\hline & $\Delta f n r$ & 115 & 135 & $1 \cdot 2$ \\
\hline & $\operatorname{arc} A$ & 125 & 1670 & $13 \cdot 4$ \\
\hline & $\operatorname{arcA} \Delta f n r$ & 100 & 43 & $0 \cdot 43$ \\
\hline
\end{tabular}

Table 4. Influence of a p6A mutation on pfl gene expression

\begin{tabular}{|c|c|c|c|c|}
\hline \multirow[t]{2}{*}{ lac $Z$ derivative } & \multirow[t]{2}{*}{ Host genotype } & \multicolumn{3}{|c|}{$\beta$-Galactosidase activity (Miller units) } \\
\hline & & $\begin{array}{l}\text { Aerobic } \\
\left(+\mathrm{O}_{2}\right)\end{array}$ & $\begin{array}{c}\text { Anaerobic } \\
\left(-\mathrm{O}_{2}\right)\end{array}$ & $\begin{array}{c}\text { Induction } \\
\text { ratio } \\
\left(-\mathrm{O}_{2} /+\mathrm{O}_{2}\right)\end{array}$ \\
\hline \multirow[t]{4}{*}{$\lambda$ RM23 (wild-type) } & wt & 455 & 5055 & $11 \cdot 0$ \\
\hline & $\Delta f n r$ & 230 & 1425 & $6 \cdot 2$ \\
\hline & $\operatorname{arcA}$ & 390 & 3630 & $9 \cdot 3$ \\
\hline & $\operatorname{arcA} \Delta f n r$ & 390 & 500 & $1 \cdot 3$ \\
\hline \multirow[t]{4}{*}{$\lambda$ RM2307 (P6A mutant) } & wt & 470 & 7510 & $16 \cdot 0$ \\
\hline & $\Delta f n r$ & 250 & 730 & $2 \cdot 9$ \\
\hline & $\operatorname{arcA}$ & 440 & 4360 & 9.9 \\
\hline & $\operatorname{arcA} \Delta f n r$ & 250 & 190 & $0 \cdot 8$ \\
\hline
\end{tabular}

\section{The influence of the $p 6 A$ promoter mutation on $p f l$ expression directed by the complete promoter system}

Table 4 shows that when promoters $P 1-P 7$ are all placed before the lac $Z$ gene in construct $\lambda R M 123$, expression was induced 11 -fold by anaerobic growth compared to the levels seen after growth in the presence of oxygen. These results are in agreement with previous findings (Sawers \& Böck, 1988, 1989; Sawers \& Suppmann, 1992; Sawers, 1993). The fnr mutation reduced the anaerobic induction ratio by approximately twofold, whilst the $\operatorname{arc} A$ mutation reduced this ratio by only $15 \%$. Only in a double null mutant was anaerobic regulation abolished. These findings indicate that both Fnr and ArcA have important roles in mediating anaerobic induction of expression when the complete promoter-regulatory region plus the downstream promoters are present.
The influence of the $p 6-2$ mutation on anaerobic and ArcA/Fnr-dependent regulation of the $p f l$ gene has been reported previously (Sawers, 1993). The p6A mutation resulted in an increase in the anaerobic induction ratio to 16 -fold in 2 RM2307 (Table 4). This is due to an increase in the level of anaerobic expression. The introduction of an fnr mutation reduced this level 10fold, while concomitantly reducing the aerobic level of expression by almost twofold. This had the consequence that the anaerobic induction ratio was reduced to threefold. The arcA mutation resulted in a $40 \%$ reduction in the anaerobic level of expression, while in the $\operatorname{arcA}$ fnr double null mutant anaerobic induction was abolished. Thus, inactivation of the P6A promoter caused an increase in the anaerobic induction ratio and resulted in expression becoming more dependent on Fnr.

That the $p 6 \mathrm{~A}$ mutation also abolished transcription 


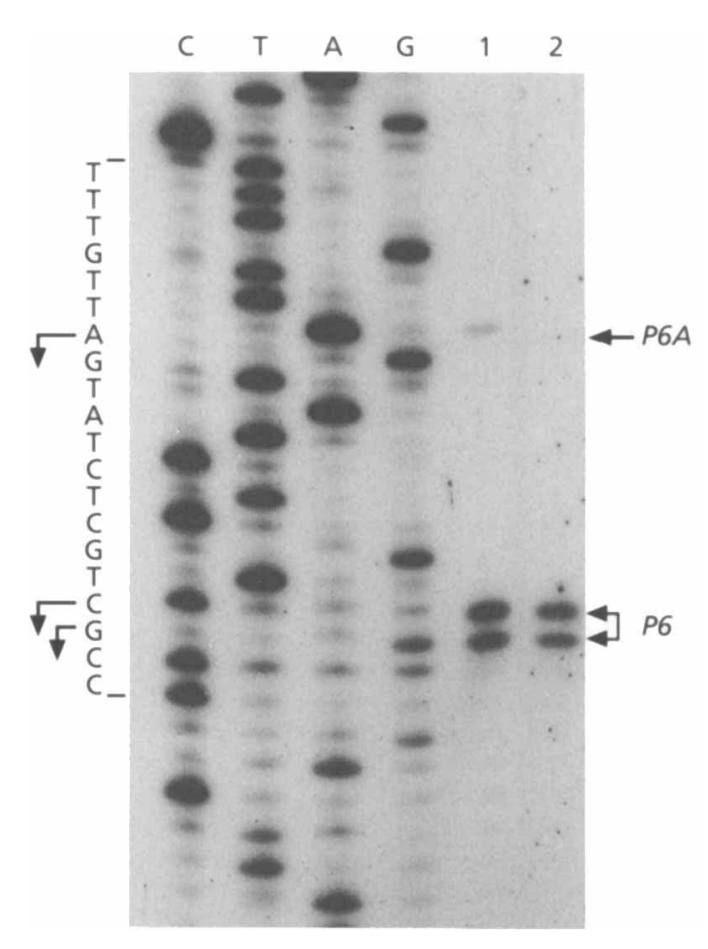

Fig. 3. Primer extension analysis of promoter $P 6$ and $P 6 A$ transcription in MC4100 and RM2307. Lanes: 1, MC4100 grown anaerobically; 2, RM2307 grown anaerobically. The initiation sites of $P 6$ and $P 6 A$ transcription are shown by arrows.

from the $P 6 A$ promoter in the derivative including all the focApfl operon promoters is demonstrated in Fig. 3. Significantly, transcription from the $P 6$ promoter was unaffected by the $p 6 A-10$ hexamer mutation. Primer extension analysis also revealed that transcription initiated with almost equal efficiency from the adjacent $C$ and $G$ residues at -1 and +1 , respectively. This is in contrast to the transcription observed with short promoter derivatives where a single initiation site from the $G$ residue at +1 was observed (Fig. 2).

\section{DISCUSSION}

\section{Overlapping P6 and P6A promoters modulate Fnr- dependent anaerobic regulation}

ArcA and Fnr control the extent of anaerobic induction of $p f l$ operon expression. The results presented in this report demonstrate that the $P 6 A$ promoter has an important function in determining the degree to which either transcription factor controls expression. The relative dependence on Fnr for anaerobic induction of expression was increased through inactivation of $P 6 A$. Although this effect was observed in three different lac $Z$ fusion constructs analysed, it was most apparent in the two sets of lac $Z$ fusions which lacked the five downstream promoters, P1-P5. Thus, the level of expression attained in anaerobically grown $p 6 \mathrm{~A}$ mutant derivatives was reduced minimally by a factor of 18 through introduction of a fnr mutation compared with only a threefold reduction in derivatives with functional $P 6 A$ promoters (see Tables 2 and 3). In the construct including all of the $p f l$ operon promoters, anaerobic expression from the $\mathrm{P}_{6} \mathrm{~A}^{+}$and $\mathrm{P} 6 \mathrm{~A}^{-}$derivatives was reduced 3.5 - and 10-fold, respectively, by the fnr mutation. In each case, anaerobic induction was abolished in $\operatorname{arcA}$ fnr double null mutants in all the derivatives tested. Therefore, although transcription from the P6A promoter is weak relative to the other $p f l$ promoters (Sawers \& Böck, 1989; Sawers, 1993), it nevertheless has a significant role in the regulation of anaerobic $p f l$ gene expression.

The major transcription initiation sites of the $P 6$ and $P 6 A$ promoters are separated by $10 \mathrm{nt}$ (Fig. 1). The $P 6$ promoter has a relatively poorly conserved -10 hexamer sequence and is lacking a -35 hexamer sequence, while the P6A promoter has a consensus -10 hexamer sequence and a poorly conserved -35 hexamer sequence. The P6A -35 hexamer sequence forms the left 'half-site' of the Fnr-binding site (Spiro \& Guest, 1990) of the P6 promoter. As a consequence, the relative affinities of RNA polymerase and Fnr for this sequence govern the frequency of transcription initiation from the $P 6$ and the P6A promoters. We noted previously that the upstream Fnr 'half-site' had one mismatch relative to the consensus Fnr-binding site (Kaiser \& Sawers, 1995). Mutation of this mismatch to create a consensus Fnr-binding site resulted in increased $P 6 A$ trancription rather than increased transcription from $P 6$. This was because the mutation also brought the -35 hexamer sequence of the P6A promoter closer to the consensus, and hence increased the relative affinity of RNA polymerase for the promoter.

\section{The requirement of $P 6 A$ activity for ArcA-dependent anaerobic regulation}

The primary effect of inactivation of the $P 6 A$ promoter was to enhance the relative dependence on Fnr for anaerobic transcriptional activation and to reduce the effective requirement for ArcA. ArcA has several binding sites in the $p f l$ promoter-regulatory region (Drapal \& Sawers, 1995a), one of which abuts the P6A -10 hexamer. Mutation of this hexamer sequence had no effect on the ability of ArcA to bind to the promoterregulatory region (data not shown). Rather, we believe that the marginally reduced dependence on ArcA for anaerobic regulation caused by the $p 6 \mathrm{~A}$ mutation (see Table 3) is probably an indirect effect due to occlusion of its function through the unimpeded binding of Fnr to its binding site at the $P 6$ promoter. Therefore, together with integration host factor (Sirko et al., 1993) and the P7 promoter (Drapal \& Sawers, 1995b), the P6A promoter has an important function in mediating ArcAdependent regulation of the focA gene.

\section{Oxygen-dependent promoter switching}

The $p f l$ operon $P 6$ and $P 6 A$ promoters provide an example of overlapping promoters where an oxygendependent switch is involved. Fnr is inactive in aero- 
bically growing cells and thus under these conditions only P6A is active. Anaerobiosis activates Fnr which results in strong transcription initiation from $P 6$ and reduced initiation from P6A. One of the best characterized examples of a switch mechanism in overlapping promoters is presented by the $P 1$ and $P 2$ promoters of the gal operon (Adhya, 1987). Much in the same way that anaerobiosis controls the switch between the $p f l P 6$ and $P 6 A$ promoters, so cAMP concentrations modulate the switch between the overlapping Crp-dependent P1 and $P 2$ promoters of the gal operon (Bingham et al., 1986; Irani et al., 1989). More recently described examples of overlapping promoters controlling anaerobic gene expression include the $P_{1}$ and $P_{2}$ promoters of the fixRnifA operon in Bradyrhizobium japonicum (Barrios et al., 1995) and the $P_{\mathrm{a}}$ and $P_{\mathrm{b}}$ promoters of the E. coli arcA gene (Compan \& Touati, 1994). The overlapping $\operatorname{arcA}$ gene promoters are of particular relevance, since, as with the $p f l$ promoters, the downstream promoter is positively regulated by Fnr. During anaerobiosis this promoter augments transcription of three constitutive promoters to increase arcA expression fourfold (Compan \& Touati, 1994).

\section{The consequences of P6A promoter activity for cellular physiology}

During aerobiosis the $P 6$ and $P 7$ promoters are inactive but the P1-P5 promoters exhibit low-level activity (Sawers \& Böck, 1989; Sawers, 1993). Consequently, the focA gene can only be transcribed by the P6A promoter aerobically, whilst the $p f l$ gene is transcribed from the $P 6 A$ promoter and the low-level activity of the P1-P5 promoters. This dependence of focA expression on $P 6 A$ promoter activity was reflected in the reduced aerobic expression observed in the operon fusion derivatives where the $l a c Z$ gene was fused with the promoterregulatory region of the focApfl operon; in contrast, inactivation of the $P 6 A$ promoter had little effect on aerobic $p f l$ expression. A further difference between foc $A$ and $p f l$ expression is the stronger dependence of focA expression on ArcA (compare Tables 3 and 4). This suggests that focA expression is induced, through ArcA, during the transition from aerobiosis through microaerobiosis, whereas $p f l$ expression may be induced to high levels at a later stage when the cells become more anaerobic. ArcA has been shown to be activated at higher redox potentials compared with Fnr (Fu et al., 1991; Tseng et al., 1996). Therefore, these differences in ArcA and Fnr dependence could introduce a temporal aspect to enzyme synthesis whereby FocA is synthesized immediately prior to Pfl. Since the FocA protein functions in regulating the intracellular formate pool (Suppmann \& Sawers, 1994), it would be of benefit to the cell to maintain low-level synthesis of FocA aerobically and to induce synthesis quickly in preparation for the shift to anaerobiosis with the concomitant rapid production of formate through the action of Pfl. Expression studies in chemostat cultures where oxygen concentration can be precisely controlled will provide experimental support for these proposals.

\section{ACKNOWLEDGEMENTS}

We thank Ray Dixon for critically reading the manuscript. This work was supported by the Deutsche Forschungsgemeinschaft and the BBSRC via a grant-in-aid to the John Innes Centre.

\section{REFERENCES}

Adhya, S. (1987). The galactose operon. In Escherichia coli and Salmonella typhimurium: Cellular and Molecular Biology, pp. 1503-1512. Edited by F. C. Neidhardt, J. L. Ingraham, K. Brooks Low, B. Magasanik, M. Schaechter \& H. E. Umbarger. Washington, DC: American Society for Microbiology.

Barrios, H., Fischer, H.-M., Hennecke, H. \& Morett, E. (1995). Overlapping promoters for two different RNA polymerase holoenzymes control Bradyrbizobium japonicum nifA expression. J Bacteriol 177, 1760-1765.

Begg, Y. A., Whyte, J. A. \& Haddock, B. A. (1977). The identification of mutants of Escherichia coli deficient in formate dehydrogenase and nitrate reductase activites using dye indicator plates. FEMS Microbiol Lett 2, 47-50.

Bingham, A. H. A., Ponnambalam, S., Chan, B. \& Busby, S. (1986). Mutations that reduce expression from the $P 2$ promoter of the Escherichia coli galactose operon. Gene 41, 67-74.

Birkmann, A., Sawers, R. G. \& Böck, A. (1987). Involvement of the $n t r A$ gene product in the anaerobic metabolism of Escherichia coli. Mol Gen Genet 210, 535-542.

Casadaban, M. J. \& Cohen, S. N. (1979). Lactose genes fused to exogenous promoters in one step using Mu-lac bacteriophage: in vivo probe for transcriptional control sequences. Proc Natl Acad Sci USA 76, 4530-4533.

Compan, I. \& Touati, D. (1994). Anaerobic activation of $\operatorname{arc} A$ transcription in Escherichia coli: roles of Fnr and ArcA. Mol Microbiol 11, 955-964.

Drapal, N. \& Sawers, G. (1995a). Purification of ArcA and analysis of its specific interaction with the $p f l$ promoter-regulatory region. Mol Microbiol 16, 597-607.

Drapal, N. \& Sawers, G. (1995b). Promoter 7 of the Escherichia coli pfloperon is a major determinant in the anaerobic regulation of expression by ArcA. J Bacteriol 177, 5338-5341.

Fu, H. A., luchi, S. \& Lin, E. C. C. (1991). The requirement of ArcA and Fnr for peak expression of the cyd operon in Escherichia coli under micro-aerobic conditions. Mol Gen Genet 226, 209-213.

Irani, M., Musso, R. \& Adhya, S. (1989). Cyclic-AMP-dependent switch in initiation of transcription from the two promoters of the Escherichia coli gal operon: identification and assay of $5^{\prime}$ triphosphate ends of mRNA by GTP:RNA guanyltransferase. J Bacteriol 171, 1623-1630.

Kaiser, M. \& Sawers, G. (1995). Fnr activates transcription from the $P 6$ promoter of the $p f$ operon in vitro. Mol Microbiol 18, 331-342.

Knappe, J. \& Sawers, G. (1990). A radical-chemical route to acetyl-CoA: the anaerobically induced pyruvate formate-lyase system of Escherichia coli. FEMS Microbiol Rev 75, 383-398.

Miller, J. H. (1972). Experiments in Molecular Genetics. Cold Spring Harbor, NY : Cold Spring Harbor Laboratory.

Sawers, G. (1993). Specific transcriptional requirements for positive regulation of the anaerobically inducible $p f l$ operon by ArcA and FNR. Mol Microbiol 10, 737-747.

Sawers, G. \& Böck, A. (1988). Anaerobic regulation of pyruvate formate-lyase from Escherichia coli K-12. J Bacteriol 170, 5330-5336. 
Sawers, G. \& Böck, A. (1989). Novel transcriptional control of the pyruvate formate-lyase gene: upstream regulatory sequences and multiple promoters regulate anaerobic expression. J Bacteriol 171, 2485-2498.

Sawers, G. \& Suppmann, B. (1992). Anaerobic induction of pyruvate formate-lyase gene expression is mediated by the ArcA and FNR proteins. J Bacteriol 174, 3474-3478.

Simons, R. W., Houman, F. \& Kleckner, N. (1987). Improved single and multicopy lac-based protein and operon fusion cloning tools. Gene 53, 85-96.

Sirko, A., Zehelein, E., Freundlich, M. \& Sawers, G. (1993). Integration host factor is required for anaerobic pyruvate induction of $p f l$ operon expression in Escherichia coli. J Bacteriol 175, 5769-5777.
Spiro, S. \& Guest, J.R. (1990). FNR and its role in oxygenregulated gene expression in Escherichia coli. FEMS Microbiol Rev 75, 399-428.

Suppmann, B. \& Sawers, G. (1994). Isolation and characterisation of hypophosphite-resistant mutants of Escherichia coli: identification of the FocA protein, encoded by the $p f l$ operon, as a putative formate transporter. Mol Microbiol 11, 965-982.

Tseng, C.-P., Albrecht, J. \& Gunsalus, R.P. (1996). Effect of microaerophilic cell growth conditions on expression of the aerobic (cyoABCDE and cydAB) and anaerobic (narGHJl, frd $A B C D$ and $d m s A B C$ ) respiratory pathway genes in Escherichia coli. J Bacteriol 178, 1094-1098.

Received 31 July 1996; revised 2 October 1996; accepted 7 October 1996. 